\title{
Mobile Tracker
}

\author{
Shirin Salim ${ }^{1}$, Dipina Damodaran $\mathrm{B}^{2}$ and Surekha Marium Vargese ${ }^{3}$ \\ Department of Computer Engineering, M A College of Engineering, Kothamangalam, \\ Kerala, India
}

\begin{abstract}
In today's fast world, mobile has become one of the important commodities of a human being. It has become a necessity rather than a luxurious commodity. The Mobile Tracking helps to track the current location of the mobile. The security of the devices powered with this is most challenging problem existing now. Almost everyone might have experienced misplacing or losing their mobile phones. Hence, it is necessary to create an app in all smart phones to detecting thefted phone. This paper discuss a tracking application including SIM detection, call monitoring, image capture, contact capture, profile switching etc for thefted mobile phone. These features are quite different from the existing tracker applications which would be helpful in tracing the lost mobile without the help of any protecting agency. The application installed will be running in the background and won't be shown in the task manager as well. Once the mobile phone is lost, this application enables the user to track a mobile device and to receive notification via SMS to a predefined number. Some specified formatted messages can be used to control the thefted mobile phone.
\end{abstract}

\section{KEYWORDS}

Mobile Tracking, GSM, GPS.

\section{INTRODUCTION}

The introduction of smartphones in the mobile market brings a tremendous change. They have many features and applications that is very useful to us. Different type of OS are available in the market, among them Android OS become widespread now a days because of its excellent features.

The Mobile Tracker, An android application to locate and track mobile phones is a unique and efficient application which has a variety of features that enhances the existing mobile tracking system [2] . The app stands different from the existing system as it is not only the GPS value it makes use of but it works on GSM/text messaging services which make it a simple and unique one. The app is able to enable the GPS when a non authorised SIM card is detected in the device by comparing the Integrated Circuit Chip Card Identification (ICC ID). The ICC ID number is unique for each SIM card. The app is filled on with features like changing profile, call monitoring, Sim card detection, and location fetching through GPS [3][4]. All these features works on SMS basis. So, incoming SMS format plays an important role. The android application running in the smartphone monitors all the incoming messages. If the SMS is received in a predefined format it reads and performs the expected task. The application installed will be running in the background and won't be shown in task manager. Many one of us have been experienced the missing of your mobile phones, if it is an android phone this application helps you to track it without the help of cyber cell. Once it is lost, this application enables the user to track a mobile device notification via SMS to a predefined number saved in that application at the time of installation itself. This application uses Android OS which demonstrates a system that uses a regular mobile phone equipped with a GPS receptor and connected to a global system for 
mobile (GSM) network that takes advantage of these technologies in behalf of the user safety [5]. The app is a useful mobile application that combines several features which aims at the user's security.

The app is directed to two user profiles, the client and server to be tracked. The server side requires any android based Smartphone starting from version Android 2.2 having the app installed in it with GPRS and GPS enabled. The client side requires any other OS based mobile phones for sending and receiving SMS. If there is any error in sending the message from the operator, there won't be any message sends to the operator by the application, instead no action takes place at the server side. This application uses Android OS which demonstrates a system that uses a regular mobile phone equipped with a GPS receptor and connected to a Global System for Mobile(GSM) network that takes advantage of these technologies in behalf of user safety. The app is a useful mobile application that combines several features which aims at the user's security.

The app is filled on with features like changing profile, call monitoring, SIM card detection, location fetching through GPS and transfer of images to email address. All these features work on the SMS basis. So, incoming SMS format plays an important role. The android application running in the smartphone running monitors all the incoming messages. If the SMS is received in a predefined format it reads the SMS it reads the SMS and performs the expected task [5][6].

The paper is organized in the following sections. Section II describes the application development including its Requirements, features and the technologies employed. Section III describes the application functionality along with experimental evaluation and results. Finally, section IV presents the conclusion and some possible future work.

\section{Problem Definition}

Almost everyone might have experienced misplacing or losing their mobile phones. Hence, it is necessary to create an app in all smart phones to detecting thefted phone. This paper discuss a tracking application including SIM detection, call monitoring, image capture, contact capture, profile switching etc for tracking mobile phone.

\subsection{ARCHITECTURE}

The working for the app contains two user profiles, one android phone starting from version 2.2 with GPS enabled where the app is installed and another is any other OS based mobile phone which is used to control the thefted android phone by sending and receiving SMS.

Figure 1 shows the Mobile Tracker requirements [4]. 


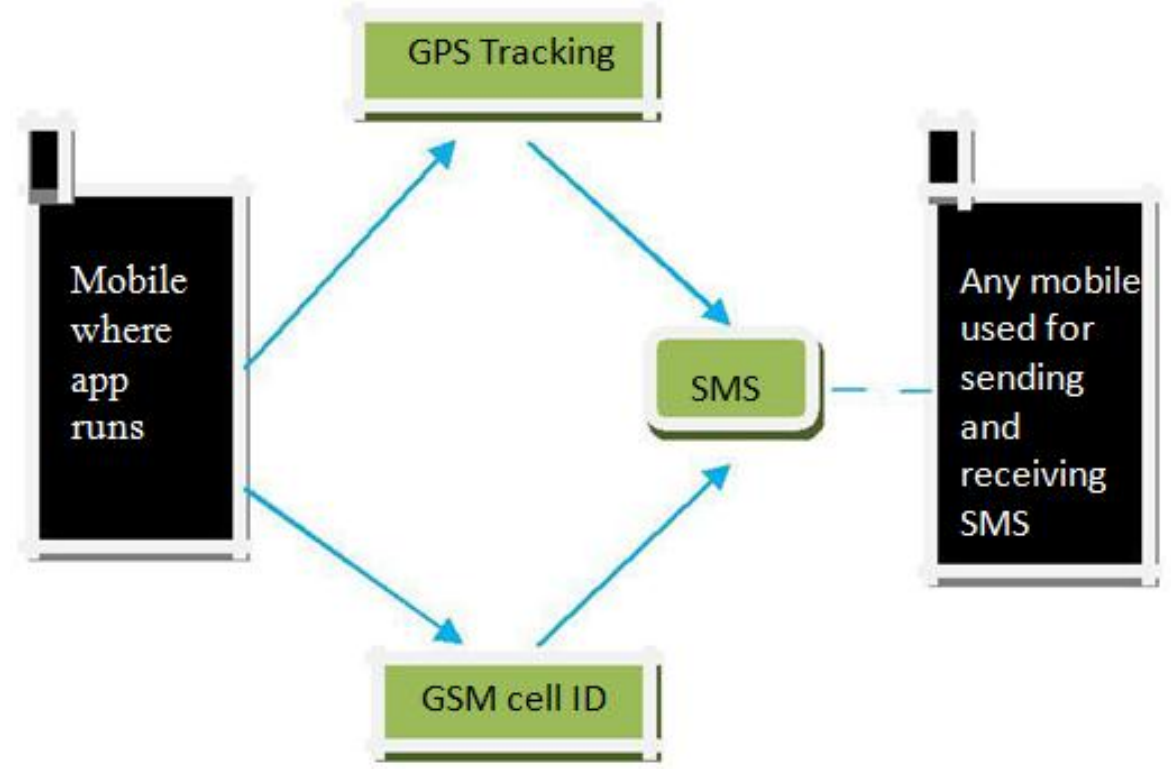

Figure 1: Mobile Tracker Architecture [1]

The SIM card detection feature of the app checks whether the currently used SIM card is authorized or not. If it detects that there is an unauthorized SIM card in the phone suddenly it will send a notification warning message to the predefined emergency number. The warning message contains the current GSM cell ID, phone number of unauthorized SIM card and also network provider.

The camera feature requires the front or back camera and internet connection on the phone.

The application fetches the location through GPS and GPS values along with address is send to the predefined number. To activate this feature the android phone must be GPS enabled. Also the phone must have the internet connection so that the details of the thefted phone can be send through email to a specified email id.

\section{Methodology}

SIM cards are identified by its Integrated Circuit Card ID(ICC-ID) and it will be stored in the SIM cards and are also get printed in the body of SIM card during the process personalization. Whenever the application starts, the ICC-ID of the current SIM card is compared with the predefined ICC-ID to detect unauthorized SIM card $\mathrm{n}$ the device. After the SIM replacement, we will get a notificaion about the IMEI number with the details of the inserted new SIM.The call history details like incoming, outgoing calls which are made from the lost antroid mobile are provided by the call monitoring feature. And also it will send all the saved numbers and name, as SMS to the predefined number. Thus we get more contact informations about the cellphone thief .Suppose the GPS data shows that the mobile is very nearer to us but we can't identify it ,so that it is in silent mode or the flicked person make the phone in silent as he is still around.Then we can convert the profile of the phone from silent to general and also from general to silent as per our requirement by just sending an SMS using the predefined format with the help of our profile management feature in the application.

By just sending a predefined SMS, we can activate the back camera of our mobile phone.whenever the message recieved ,the application activates the back camera and capture image and send it to our email ID auomatically that we had saved in the application earlier. And 
we can check our email account ,thus we can extract more information about the thief like who, how, where etc. Using a particular predefined format SMS we can also find out or retrieve the current location of the lost Smart phone. Whenever the SMS get we can retieve the current location of the thefted phone at any time. Also we can retrieve all the inbox details, that is messages that the thief or new owner gets to the predefined number by sending another format of message. Thus we can get more information about his friends and all. If we want to block the thief to making use of our Smart phone that he thefted, we can block him by sending an SMS to the predefined number, so that he cant make call enough. Also we can remove the block whenever we want.

The main advantage of the application is that it automatically deletes the incoming and outgoing SMS from the Smart phone that is thefted as it get installed the app such that the new owner or the thief who using the cell is clueless about it all.

The following is the data flow diagram of the Mobile Tracker application:

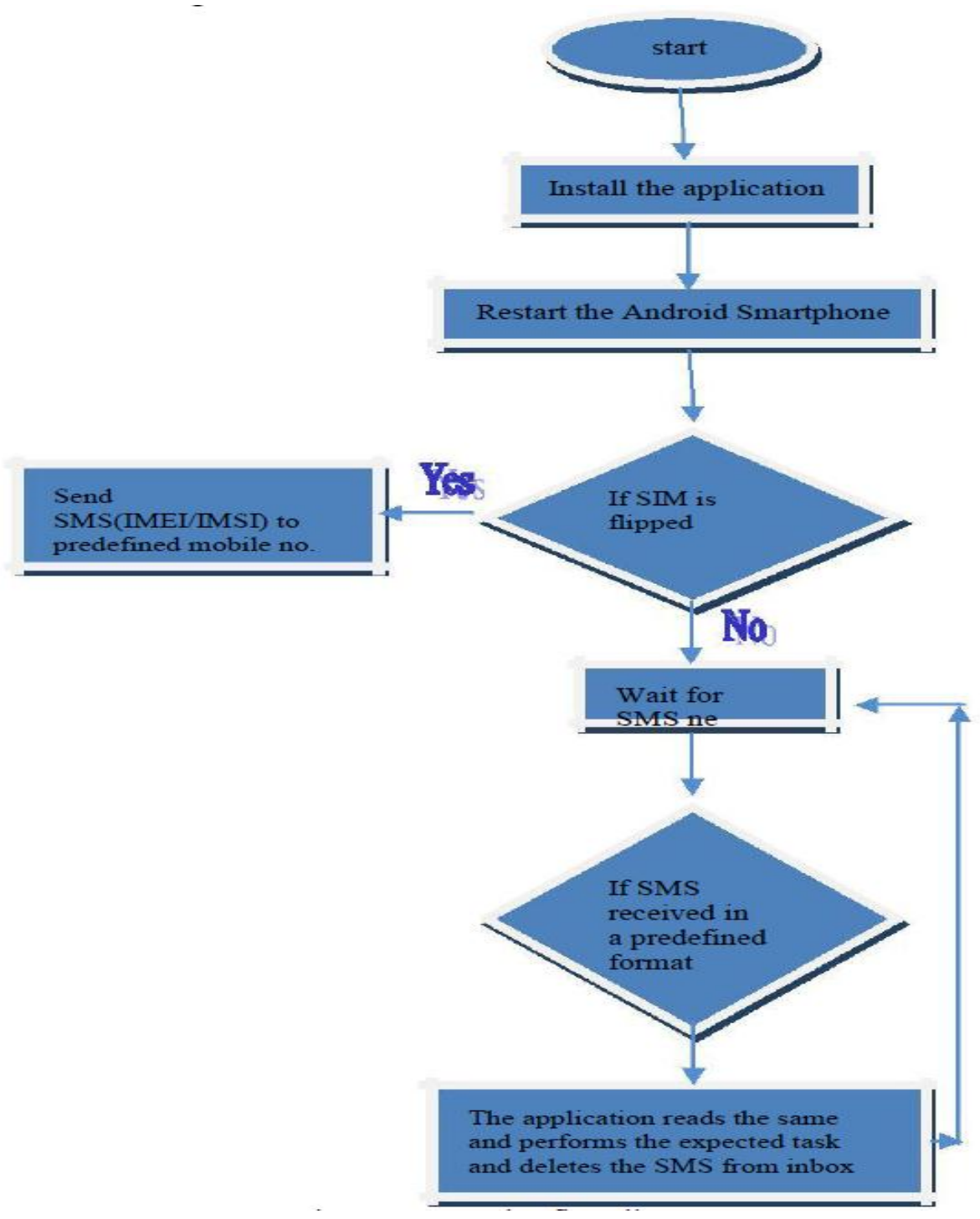

Figure 2. Dataflow Diagram[1] 


\section{Algorithm:}

Step1:Start the process.

Step2:Install the application. After installing athe application on the Smart phone, it will be set to start running in background every time the device os restarts.

Step3:Restart the Antroid Smart phone.

Step4:If the SIM is flipped the application sends SMS regarding the details of the new SIM to the predefined mobile no.

Step5:The application auto starts every time the mobile boots up. Then it goes to running mode and will start the main service which continuously listens for the incoming SMS messages.

Step6:Whenever it gets a new SMS ,it checks the content of the message and if the message is in a particular format, application reads the same ,performs the expected task and replies back to the previously number.

The application installed will be running in the background and won't be shown in the task manager as well. Once the mobile phone is lost ,this application enables the user to track a mobile device and to receive notification via SMS to a predefined number.

\section{EXPERIMENTAL RESULTS}

User is the person who sends the message to the lost android phone using the application.

1. If SIM is replaced the predefined number will receive the IMEI/IMSI number and details of the newly inserted SIM.

2. If SMS is of the format @ tracklocation then can find out the location of the thefted phone.

3. If SMS is of the format @ trackcontacts then can findout the contacts of the thefted phone.

4. If SMS is of the format @ trackcall log then can find out the call logs of the thefted phone.

5. If SMS is of the format @ trackmessages then can find out the messages which are there in the thefted phone

6. If SMS is of the format @ trackSR then can convert SILENT mode to the RING mode.

7. If SMS is of the format @ trackRS then can convert RING mode to SILENT mode.

8.If SMS is of the format @ trackactive then can convert into the blocking mode.

9.If SMS is of the format @ tracknonactive then can convert into the nonblocking mode.

10. If SMS is of the format @ trackpicture then can take the picture of the person.

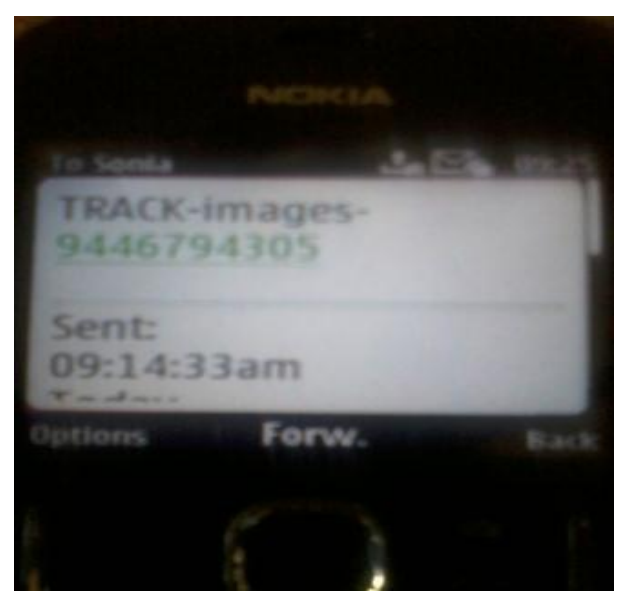

Figure 3. sim change happens 
International Journal on Cybernetics \& Informatics (IJCI) Vol. 5, No. 2, April 2016
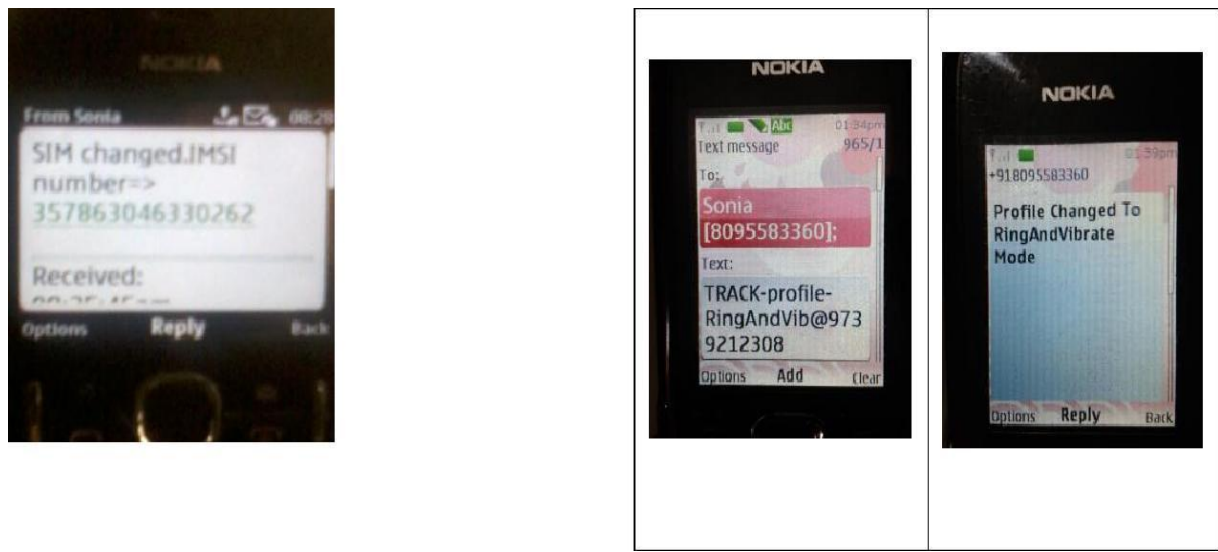

Figure 4.capturing imag
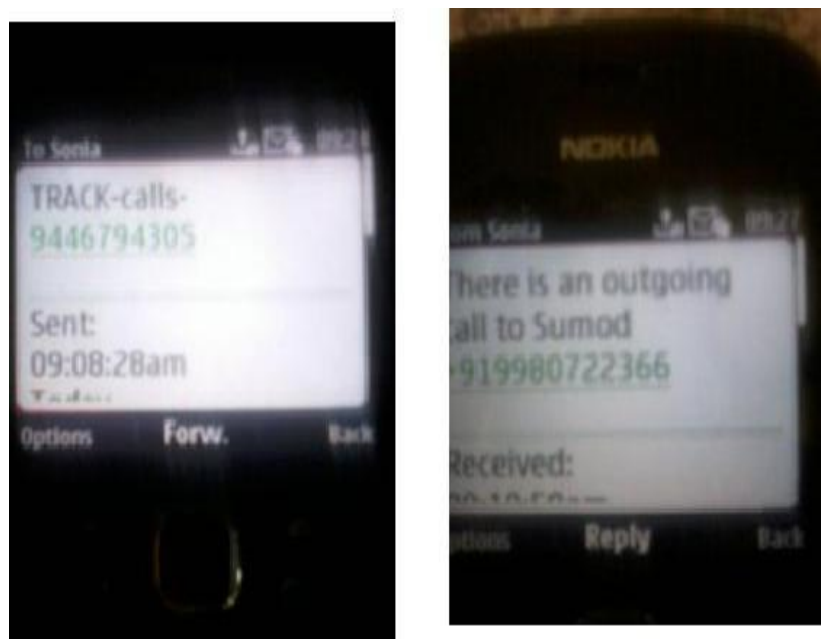

Figure 5: capturing incoming and outgoing calls

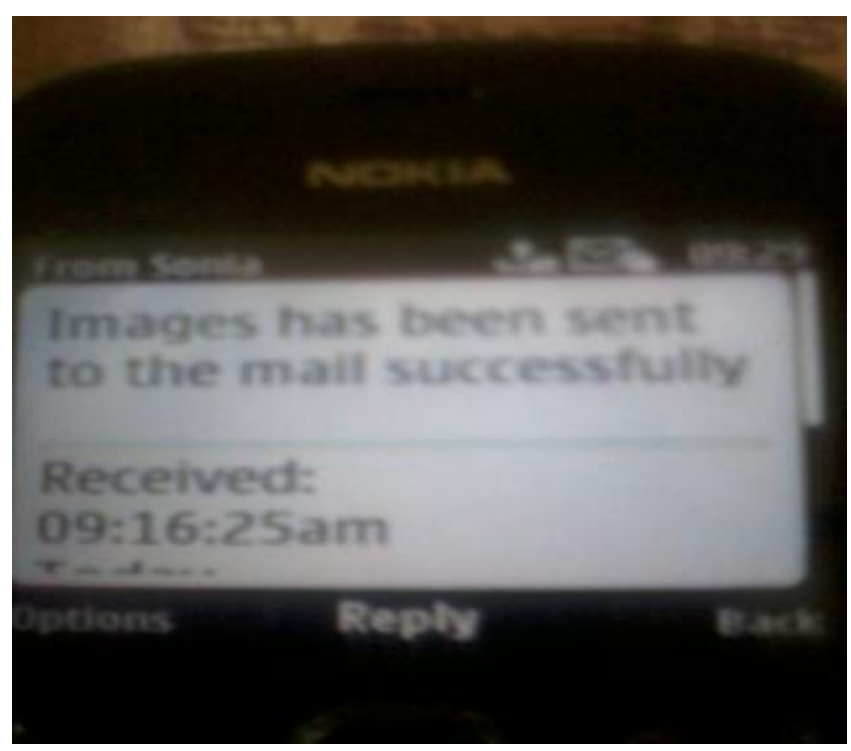

Figure 6. mail image to address 


\section{CONCLUSiON}

The mobile tracker is an efficient application for tracking of mobile phone. It uses the GPS and WI-FI for finding the location. When the SIM is replaced it will detect the location, track picture, call $\log$, call details, and profile changing-silent to ring and ring to silent, track picture. This application doesn't work if the phone is switched off. For future work, it is proposed to implement some algorithm where the phone itself identifies that it is being lost. Whenever, the phone is off for more than 48 hours it should make it switch on automatically.

\section{REFERENCES}

[1] Alzantot, M. , Youssef, M. "UPTIME: Ubiquitous pedestrian tracking \& locating using mobile phones "Wireless Res. Center, Egypt-Japan Univ. of Sc. \& Tech. (E-JUST), Alexandria, Egypt Sonia C.V, Dr. A.R.Aswatha M.Tech.(student), Telecommunication Engineering Department DSCE, VTU, Bangalore, India Professor \& Head of the Department (Telecommunication Engineering) DSCE, VTU, Bangalore, India.

[2] Luis C.M Varandas;Binod Vaidya;Joel J.P.C Rodrigues; "mTracker: A Mobile Tracking Application for Pervasive Environment" IEEE 24th International Conference on Advanced Information Networking and Applications Workshops,pp.962-967April 2010.

[3] Lin, Ding-Bing B. "Mobile location estimation and tracking for GSM systems" IEEE 15th International Conference on Personal, Indoor and Mobile Radio Communications, vol.4, pp.28352839, Sep. 2004.

[4] Bayir, Murat Ali" Track me! a web based location tracking and analysis system for smart phone users" 24th International Symposium on Computer and Information Sciences, pp.117-122,Sep.2009.

[5] Sangwoo Cho; Haekyung Jwa; Joohwan Chun; Jong Heun Lee; Yoon Seok Jung; "Mobile position location with the constrained bootstrap filter in a cellular communicationsystem"Thirty-Fourth Asilomar Conference $\mathrm{n}$ the context of near field communication applications", International Conference on Management of Mobile business, 2007, p.5.

[6] Madlmayr, G.; Dillinger, O.; Langer, J.; Schaffer, C.; Kantner, C. "The benefit of using SIM application toolkit i[5]Hellebrandt,Martin ,Mathar,Rudolf "Location tracking of mobiles in cellular radio networks" IEEE Transactions on Vehicular Technology,vol.48,pp.1558-1562,Sep1999.

\section{AUTHORS}

Shirin Salim. is currently pursuing M.Tech in Computer Science and Engineering in Mar Athanasius College of Engineering. She completed her B.Tech from Ilahia college of Engineering and Technology, Muvattupuzha. Her areas of research are Machine Learning and Data Mining.

Dipina Damodaran B is currently pursuing M.Tech in Computer Science and Engineering in Mar Athanasius College of Engineering. She completed her B.Tech from Malabar College of Engineering Trissur. Her areas of research are Networking, Data Structures and Data Mining.

Surekha Mariam Varghese is currently heading the Department of Computer Science and Engineering, M.A. College of Engineering, Kothamangalam, Kerala, India. She received her B-Tech Degree in Computer Science and Engineering in 1990 from College of Engineering, Trivandrum affiliated to Kerala University and M-Tech in Computer and Information Sciences from Cochin University of Science and Technology, Kochi in 1996. She obtained Ph.D in Computer Security from Cochin University of Science and
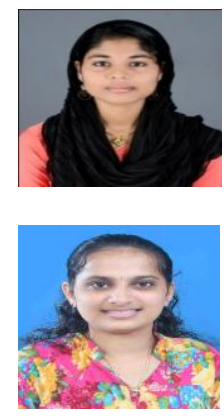
Technology, Kochi in 2009. She has around 25 years of teaching and research experience in various institutions in India. Her research interests include Network Security, Machine Learning Database Management, Data Structures and Algorithm. 